\title{
Trichloroisocyanuric Acid: An Alternate Green Route for the Transformation of Alkenes into Epoxides
}

\author{
Mira Wengert, Antonio M. Sanseverino and Marcio C. S. de Mattos* \\ Instituto de Química, Universidade Federal do Rio de Janeiro, CP 68545, 21945-970 Rio de Janeiro - RJ, Brazil
}

\begin{abstract}
Epóxidos podem ser preparados eficientemente a partir da reação de alquenos com ácido tricloroisocianúrico em acetona aquosa seguido de tratamento da cloroidrina resultante com $\mathrm{KOH}$ aquoso em éter / pentano.
\end{abstract}

The preparation of epoxides is efficiently achieved in mild conditions by reaction of alkenes with trichloroisocyanuric acid in aqueous acetone followed by treatment of the resulting chlorohydrin with aqueous $\mathrm{KOH}$ in ether / pentane.

Keywords: chlorohydrin, alkene, epoxide, trichloroisocyanuric acid, green chemistry

\section{Introduction}

Epoxides are useful compounds in synthetic organic chemistry. ${ }^{1}$ There are several methodologies for the preparation of these compounds and among them, the oxidation of alkenes with peracids and the cyclization of halohydrins with bases are the most employed. ${ }^{1}$ Peracids and analogues have been used to prepare epoxides, even though these reagents must be manipulated with care, due to their tendency to explosion. ${ }^{2}$ Halohydrins have also found wide use in epoxide synthesis in both laboratorial and industrial scale, ${ }^{3}$ and the halogenation of alkenes in dilute aqueous solutions is an effective way to prepare these intermediates. ${ }^{4}$ Although this methodology is effective, the handling and manipulation of toxic and corrosive halogens is problematic, specially in large-scale. Nowadays, the utilization of health, safe, and readily available materials is very attractive and consistent with green chemistry challenges. ${ }^{5}$

Trichloroisocyanuric acid [1,3,5-trichoro-1,3,5triazine-2,4,6- $(1 \mathrm{H}, 3 \mathrm{H}, 5 \mathrm{H})$-trione, $(\mathbf{1})]$ is a stable and inexpensive solid frequently found in a large number of commercial products for swimming-pool disinfection ${ }^{6}$ and easily available in pool supply and some hardware stores. Recent examples of the utilization of trichloroisocyanuric acid in organic synthesis are thioacetalization of carbonyl and related compounds, ${ }^{7}$ conversions of alcohols to halides and carboxylic acids to acid chlorides, ${ }^{8}$ and oxidations of

* e-mail: mmattos@iq.ufrj.br alcohols to carbonyl compounds, ${ }^{9}$ aldoximes to nitrile oxides,,$^{10}$ thiols to disulfides, ${ }^{11}$ selenols to diselenides, ${ }^{12}$ and sulfides to sulfoxides. ${ }^{13}$

The decomposition of $\mathbf{1}$ in water produces hypochlorous acid, ${ }^{14}$ that can react with alkenes to produce chlorohydrins. So, we communicate here our initial results on the reaction of alkenes with trichloroisocyanuric acid to produce chlorohydrins and theirs base conversion to epoxides.

\section{Results and Discussion}

The reactions were carried out stirring together $10 \mathrm{mmol}$ of cyclohexene with $4 \mathrm{mmol}$ of $\mathbf{1}$ in diverse solvents at room temperature and the results are shown in Scheme 1. The best results were obtained with aqueous acetone, which produced trans-2-chlorocyclohexanol in almost quantitative chromatographic yield in only 30 min reaction time. Water or aqueous acetonitrile produced a mixture where the chlorohydrin predominated and using a biphasic system (dichloromethane or ethyl acetate) it was obtained several products or the reaction was incomplete after several hours.

The formation of all products could easily be rationalized in terms of the electrophilic chlorination of cyclohexene by the intermediacy of hypochlorous acid to produce a chloronium ion followed by nucleophilic participation of the solvent. Interestingly, the formation of trans-1,2-dichlorocyclohexane in the reaction of cyclohexene with $\mathbf{1}$ in pure water could be rationalized by acid-decompositon of hypochlorous acid to chlorine. 
<smiles>CC(O)C1(O)CCCC[C@@H]1O</smiles>

(1)<smiles>CC(=O)NC1CCCCC1Cl</smiles><smiles>CC(=O)C[C@H](C)O</smiles>

$95 \%$

Scheme 1.

Based on the above results, 1-hexene, 1-octene, 1methylcyclohexene, styrene, and $\alpha$-methylstyrene reacted with $\mathbf{1}$ in aqueous acetone to produce the corresponding chlorohydrins (> $80 \%$ purity by HRGC). In general, the regioselectivity was very high and no regioisomeric products were detected by the analytical methods employed. Exceptions were the aliphatic monosubstituted alkenes 1-hexene and 1-octene that afforded a regioisomeric mixture of chlorohydrins in wich the 1chloro-2-alkanols predominated (ca. $3: 1$ by HRGC). 1Methylcyclohexene produced a very unstable chlorohydrin which was immediately converted to the epoxide (vide infra).

The crude chlorohydrins were treated with $\mathrm{KOH}$ in a biphasic media (water / ether / pentane) to produce the epoxides in excellent total isolated yields (Scheme 2). The purity of the products was $>90-99 \%$ (by HRGC) and they were characterized by ${ }^{1} \mathrm{H}$ and ${ }^{13} \mathrm{C}$ NMR, MS, and HRGC (coelution with authentic samples).

Disappointing results were obtained when the methodology was applied to limonene as it was produced trans-1,2-epoxylimonene ( $c a .45 \%$ by HRGC, confirmed

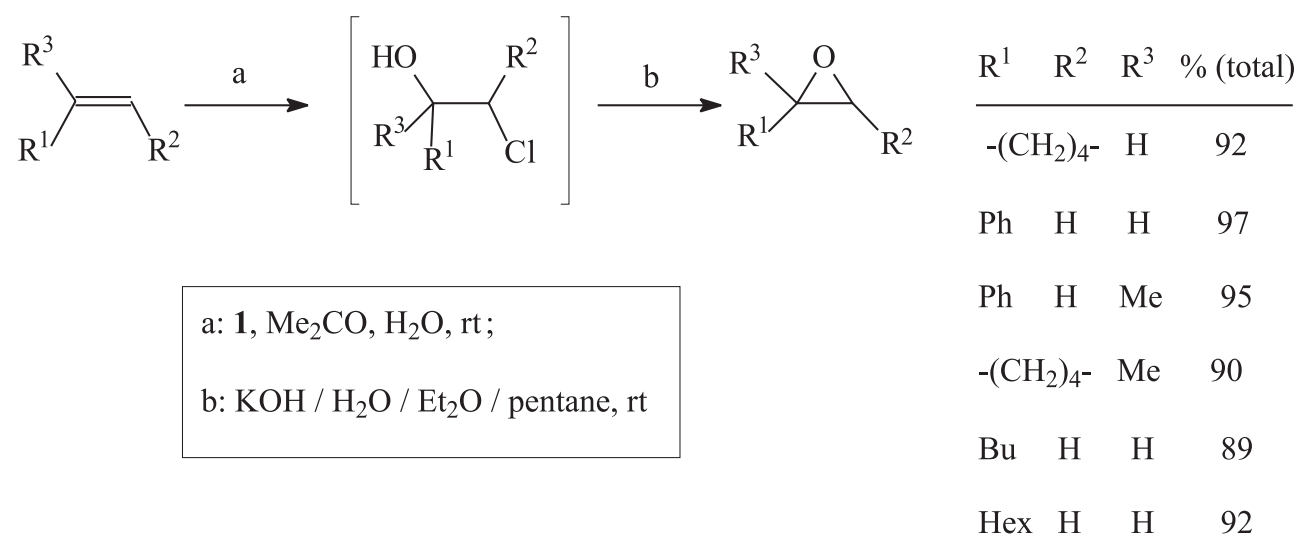


by coinjection with an authentic sample ${ }^{15}$ ) along with unreacted substrate and several other minor products (none of them limonene epoxides) Scheme 3.

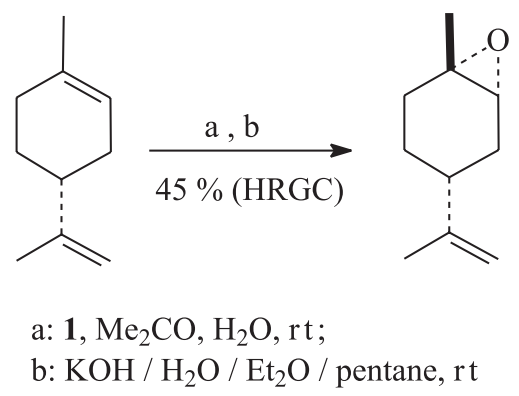

Scheme 3 .

In summary, our methodology is an alternate route for the preparation of epoxides from alkenes in high yields. The reactions are simple, safe, and all the reagents are cheap and readily available, which are in accordance with the green chemistry philosophy. ${ }^{16}$

\section{Experimental}

All chemicals were used without further purification. ${ }^{1} \mathrm{H}$ and ${ }^{13} \mathrm{C}$ NMR were acquired on a Bruker AC-200 (200 and $50 \mathrm{MHz}$, respectively) spectrometer in $\mathrm{CDCl}_{3}$ solutions with TMS as internal standard. Analyses by HRGC were performed on a HP-5890-II gas chromatograph with FID by using a $28 \mathrm{~m}$ (length), $0.25 \mathrm{~mm}$ (ID) and $0.50 \mathrm{~mm}$ (phase thickness) RTX-5 silica capillary column and He (flow rate $50 \mathrm{~cm} \mathrm{~s}^{-1}$ ) as carrier gas (split $1: 10$ ). Mass spectra were obtained on a Hewlett-Packard HP 5896-A HRGC-MS using electron impact $(70 \mathrm{eV})$.

\section{Typical procedure for the preparation of chlorohydrins}

To a stirred solution of the alkene $(10 \mathrm{mmol})$ in a solution $\left(30 \mathrm{~cm}^{3}\right)$ of water/acetone (1: 5), trichloroisocyanuric acid (4 mmol) was added at $\mathrm{rt}$ in small portions. After $30 \mathrm{~min}$, the suspension was filtered, $\mathrm{CHCl}_{3}$ $\left(20 \mathrm{~cm}^{3}\right)$ was added and the resulting solution was washed with $10 \% \mathrm{NaHSO}_{3}$ and water. The organic layer was dried (anhydrous $\mathrm{Na}_{2} \mathrm{SO}_{4}$ ) and analysed by HRGC. Evaporation of the solvent on a rotatory evaporator gave the crude chlorohydrins.

Trans-2-chlorocyclohexanol. ${ }^{1} \mathrm{H}$ NMR: $\delta$ 1.05-2.84 (m, 8H), 2.60 (broad s, 1H, OH), 3.30 (s, 1H), 3.39 (s, 1H) ppm. ${ }^{13} \mathrm{C} \mathrm{NMR:} \delta 24.0\left(\mathrm{CH}_{2}\right), 25.6\left(\mathrm{CH}_{2}\right), 33.1\left(\mathrm{CH}_{2}\right), 35.1\left(\mathrm{CH}_{2}\right)$, $67.3(\mathrm{CH}), 75.2(\mathrm{CH})$ ppm. MS: m/z 57 (100\%), 80, 98, 116, $134\left(\mathrm{M}^{+}, 6 \%\right), 136\left(\mathrm{M}+2^{+}, 2 \%\right)$. 2-chloro-1-phenylethanol. ${ }^{1} \mathrm{H}$ NMR: $\delta 2.69(1 \mathrm{H}$, broad s, OH), 3.67 (dd, $J 11.2 \mathrm{~Hz}$ and $7.9 \mathrm{~Hz}, 1 \mathrm{H}), 3.72(\mathrm{dd}, J 11.2$ $\mathrm{Hz}$ and $4.4 \mathrm{~Hz}, 1 \mathrm{H}), 4.88$ (dd, J 7.9 Hz and $4.4 \mathrm{~Hz}, 1 \mathrm{H}), 7.35$ $(\mathrm{m}, 5 \mathrm{H}) \mathrm{ppm} .{ }^{13} \mathrm{C}$ NMR: $\delta 50.5\left(\mathrm{CH}_{2}\right), 74.1(\mathrm{CH}), 126.0$ $(\mathrm{CH}), 128.4(\mathrm{CH}), 129.0(\mathrm{CH}), 140.1$ (C) ppm.

1-chloro-2-phenyl-2-propanol. ${ }^{1} \mathrm{H} \mathrm{NMR}: \delta 1.61$ (s, 3H), 2.70 (broad s, 1H, OH), 3.72 (d, J11.2 Hz, 1H), 3.81 (d, $J$ $11.2 \mathrm{~Hz}, 1 \mathrm{H}), 7.63(\mathrm{~m}, 5 \mathrm{H}) \mathrm{ppm} .{ }^{13} \mathrm{C} \mathrm{NMR}: \delta 25.1\left(\mathrm{CH}_{3}\right)$, $55.4\left(\mathrm{CH}_{2}\right), 73.9(\mathrm{C}), 124.8(\mathrm{CH}), 127.6(\mathrm{CH}), 128.9(\mathrm{CH})$, 144.3 (C) ppm.

1-chloro-2-hexanol. ${ }^{1} \mathrm{H}$ NMR: $\delta 0.90(\mathrm{~m}, 3 \mathrm{H}), 1.10-$ 1.80 (m, 6H), 2.25 (broad d, J 4.4 Hz, 1H, OH), 3.50 (dd, J 10.9 and $6.4 \mathrm{~Hz}, 1 \mathrm{H}), 3.60$ (dd, $J 10.9$ and $3.2 \mathrm{~Hz}, 1 \mathrm{H}), 3.80$ $(\mathrm{m}, 1 \mathrm{H})$ ppm. ${ }^{13} \mathrm{C}$ NMR: $\delta 14.0\left(\mathrm{CH}_{3}\right), 22.6\left(\mathrm{CH}_{2}\right), 27.7$ $\left(\mathrm{CH}_{2}\right), 34.0\left(\mathrm{CH}_{2}\right), 50.5(\mathrm{CH}), 71.5\left(\mathrm{CH}_{2}\right) \mathrm{ppm}$.

1-chloro-2-octanol. ${ }^{1} \mathrm{H}$ NMR: $\delta 0.88$ (m, 3H), 1.30-2.00 (m, 10H), 2.20 (broad d, J 4.4 Hz, 1H, OH), 3.46 (dd, J 11.0 and $7.0 \mathrm{~Hz}, 1 \mathrm{H}), 3.62(\mathrm{dd}, J 11.0$ and $3.3 \mathrm{~Hz}, 1 \mathrm{H}), 3.80(\mathrm{~m}, 1 \mathrm{H})$ ppm. ${ }^{13} \mathrm{C}$ NMR: $\delta 14.0\left(\mathrm{CH}_{3}\right), 22.6\left(\mathrm{CH}_{2}\right), 25.5\left(\mathrm{CH}_{2}\right), 29.2$ $\left(\mathrm{CH}_{2}\right), 31.8\left(\mathrm{CH}_{2}\right), 34.2\left(\mathrm{CH}_{2}\right), 50.1(\mathrm{CH}), 71.4\left(\mathrm{CH}_{2}\right) \mathrm{ppm}$.

Typical procedure for the preparation of epoxides

A biphasic solution of the crude chlorohydrin in $\mathrm{Et}_{2} \mathrm{O}$ $\left(10 \mathrm{~cm}^{3}\right)$, pentane $\left(10 \mathrm{~cm}^{3}\right), \mathrm{KOH}(11 \mathrm{mmol})$, and water $\left(6 \mathrm{~cm}^{3}\right)$ was stirred at rt. After $1 \mathrm{~h}$, the organic layer was separated, washed with water and dried (anhydrous $\mathrm{Na}_{2} \mathrm{SO}_{4}$ ). Evaporation of the solvent on a rotatory evaporator gave the pure epoxides.

Cyclohexene oxide. ${ }^{~} \mathrm{H}$ NMR: $\delta$ 1.37-1.16 (m, 4H), 1.87$1.74(\mathrm{~m}, 4 \mathrm{H}), 3.05$ (s, 2H), ppm. ${ }^{13} \mathrm{C} \mathrm{NMR}: \delta 19.4\left(\mathrm{CH}_{2}\right)$, $24.2\left(\mathrm{CH}_{2}\right), 55.1(\mathrm{CH}) \mathrm{ppm}$. MS: $m / z$ 69, 83 (100\%), 97, 98 $\left(\mathrm{M}^{+}, 3 \%\right)$.

Styrene oxide. ${ }^{1} \mathrm{H}$ NMR: $\delta 2.79(\mathrm{dd}, J 5.5 \mathrm{~Hz}$ and $2.6 \mathrm{~Hz}$, 1H), 3.13 (dd, $J 5.5 \mathrm{~Hz}$ and $4.0 \mathrm{~Hz}, 1 \mathrm{H}), 3.86$ (dd $J 2.6 \mathrm{~Hz}$ and $4.0 \mathrm{~Hz}, 1 \mathrm{H}), 7.34(\mathrm{~m}, 5 \mathrm{H}) \mathrm{ppm} .{ }^{13} \mathrm{C} \mathrm{NMR}: \delta 51.3\left(\mathrm{CH}_{2}\right)$, $52.5(\mathrm{CH}), 125.6(\mathrm{CH}), 128.3(\mathrm{CH}), 128.9(\mathrm{CH}), 137.7(\mathrm{C})$ ppm. MS: $m / z, 54,89,90,91(100 \%), 119,120\left(\mathrm{M}^{+}, 30 \%\right)$.

$\alpha$-methylstyrene oxide. ${ }^{1} \mathrm{H}$ NMR: $\delta 1.69$ (s, 3H), 2.92 $(\mathrm{d}, 1 \mathrm{H}), 3.00(\mathrm{~d}, 1 \mathrm{H}), 7.30(\mathrm{~m}, 5 \mathrm{H}), \mathrm{ppm} .{ }^{13} \mathrm{C}$ NMR: $\delta 21.9$ $\left(\mathrm{CH}_{3}\right), 56.9\left(\mathrm{CH}_{2}\right), 57.1(\mathrm{CH}), 124.9\left(\mathrm{CH}_{2}\right), 127.6\left(\mathrm{CH}_{2}\right)$, $128.5\left(\mathrm{CH}_{2}\right), 141.3$ (C) ppm. MS: m/z 78, 103, 105, 106, $133(100 \%), 134\left(\mathrm{M}^{+}, 20 \%\right)$.

1-methylcyclohexene oxide. ${ }^{1} \mathrm{H}$ NMR: $\delta$ 0.94-1.20 (m, 
$2 \mathrm{H}), 1.22(\mathrm{~s}, 3 \mathrm{H}), 1.31-1.36(\mathrm{~m}, 2 \mathrm{H}), 1.59(\mathrm{~m}, 2 \mathrm{H}), 1.78-$ $1.82(\mathrm{~m}, 2 \mathrm{H}), 2.87$ (broad s, $1 \mathrm{H}) \mathrm{ppm} .{ }^{13} \mathrm{C}$ NMR: $\delta 19.7$ $\left(\mathrm{CH}_{3}\right), 20.1\left(\mathrm{CH}_{2}\right), 22.7\left(\mathrm{CH}_{2}\right), 25.0\left(\mathrm{CH}_{2}\right), 29.9\left(\mathrm{CH}_{2}\right), 57.8$ (CH), 59.6 (C) ppm. MS: m/z 43, 55, 97 (100\%), 116, 112 $\left(\mathrm{M}^{+}, 5 \%\right)$.

1-hexene oxide. ${ }^{1} \mathrm{H}$ NMR: $\delta$ 0.82-0.87 (t, 3H), 1.32-1.47 $(\mathrm{m}, 6 \mathrm{H}), 2.38(\mathrm{dd}, J 8.0$ and $2.7 \mathrm{~Hz}, 1 \mathrm{H}), 2.66(\mathrm{dd}, J 8.0$ and $2.1 \mathrm{~Hz}, 1 \mathrm{H}), 2.83(\mathrm{~m}, 1 \mathrm{H}) \mathrm{ppm} .{ }^{13} \mathrm{CNMR}: \delta 14.1\left(\mathrm{CH}_{3}\right), 22.1$ $\left(\mathrm{CH}_{2}\right), 26.8\left(\mathrm{CH}_{2}\right), 31.7\left(\mathrm{CH}_{2}\right), 47.2\left(\mathrm{CH}_{2}\right), 52.5(\mathrm{CH}) \mathrm{ppm}$.

1-octene oxide. ${ }^{1} \mathrm{H}$ NMR: $\delta 0.88(\mathrm{t}, 3 \mathrm{H}), 1.20-1.55$ (m, $10 \mathrm{H}), 2.45(\mathrm{dd}, J 8.2$ and $3.9 \mathrm{~Hz}, 1 \mathrm{H}), 2.75$ (dd, $J 8.2$ and $1.1 \mathrm{~Hz}, 1 \mathrm{H}), 2.98(\mathrm{~m}, 1 \mathrm{H}) \mathrm{ppm} .{ }^{13} \mathrm{C}$ NMR: $\delta 14.0\left(\mathrm{CH}_{3}\right)$, $22.6\left(\mathrm{CH}_{2}\right), 26.1\left(\mathrm{CH}_{2}\right), 29.2\left(\mathrm{CH}_{2}\right), 31.8\left(\mathrm{CH}_{2}\right), 32.5\left(\mathrm{CH}_{2}\right)$, $47.0\left(\mathrm{CH}_{2}\right), 52.4(\mathrm{CH}) \mathrm{ppm}$.

\section{Acknowledgements}

MW and AMS thank PIBIC/UFRJ and CNPq, respectively, for fellowships. We thank Joel Jones Jr. and W. Bruce Kover for helpful discussions.

\section{References}

1. Smith, J.G.; Synthesis 1984, 629; Rao, A.S.; Paknikar, S.K.; Kirtane, J.G.; Tetrahedron 1983, 39, 2323.

2. Bretherick, L.; Hazards in the Chemical Laboratory, $4^{\text {th }}$ ed., Royal Society of Chemistry: London, 1986, p 446-447.

3. Boguslavskaya, L.S.; Russ. Chem. Rev. 1972, 41, 740; Sanseverino, A.M.; de Mattos, M.C.S.; Synth. Commun. 1998,
28, 559; Weissermel, K.; Arpe, H.-J; Industrial Organic Chemistry, $3^{\text {rd }}$ ed., VCH: Wienheim, 1997, p 266-267.

4. Cornforth, J.W.; Green, D.T.; J. Chem. Soc. C 1970, 846; de Mattos, M.C.S.; Sanseverino, A.M.; J. Chem. Res. (S) 1994, 440; Sanseverino, A.M.; de Mattos, M.C.S.; Synthesis 1998, 1584 .

5. Clark, J.H.; Green Chem. 1999, 1, 1; Sanseverino, A.M.; Quim. Nova 2000, 23, 102.

6. Hiegel, G.A.; Nalbandy, M.; Synth. Commun. 1992, 22, 1589; Walters, T.R.; Zajac Jr., W.W.; Woods, J.M.; J. Org. Chem. 1991, 56, 316.

7. Firouzabadi, H.; Iranpoor, N.; Hazarkhani, H.; Synlett 2001, 1641.

8. Hiegel, G.A.; Ramirez, J.; Ban, R.K.; Synth. Commun. 1999, $29,1415$.

9. De Luca, L.; Giacomelli, G.; Porcheddu, A.; Org. Lett. 2001, 3, 3041.

10. Rodrigues, R.D.; de Aguiar, A.P.; Synth. Commun. 2001, 31, 3075 .

11. Zhong, P.; Guo, M.P.; Synth. Commun. 2001, 31, 1589.

12. Zhong, P.; Guo, M.P.; Synth. Commun. 2001, 31, 1507.

13. Xiong, Z.X.; Huang, N.P.; Synth. Commun. 2001, 31, 245.

14. Juenge, E.C.; Spangler, P.L.; Duncan, W.P.; J. Org. Chem. 1966, 31, 3836.

15. Sanseverino, A.M.; da Silva, F.M.; Jones Jr., J.; de Mattos, M.C.S.; J. Braz. Chem. Soc. 2000, 11, 381.

16. Anastas, P.T.; Warner, J.C.; Green Chemistry: Theory and Practice, Oxford University Press: Oxford, 2000.

Received: January 4, 2002 Published on the web: August 28, 2002 